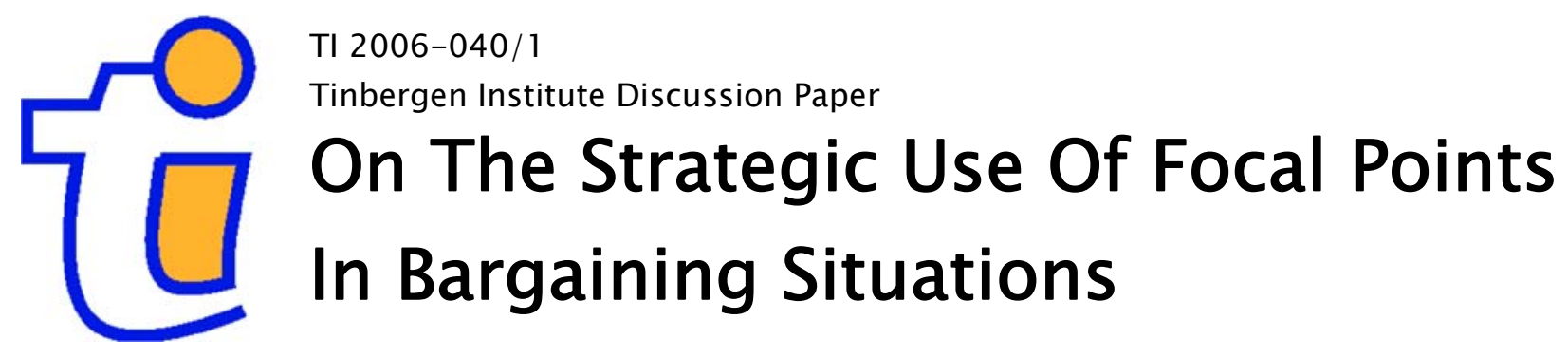

Maarten C.W. Janssen

Department of Economics, Erasmus Universiteit Rotterdam, and Tinbergen Institute. 


\section{Tinbergen Institute}

The Tinbergen Institute is the institute for economic research of the Erasmus Universiteit Rotterdam, Universiteit van Amsterdam, and Vrije Universiteit Amsterdam.

Tinbergen Institute Amsterdam

Roetersstraat 31

1018 WB Amsterdam

The Netherlands

Tel.: $\quad+31(0) 205513500$

Fax: $\quad+31(0) 205513555$

Tinbergen Institute Rotterdam

Burg. Oudlaan 50

3062 PA Rotterdam

The Netherlands

Tel.: $\quad+31(0) 104088900$

Fax: $\quad+31(0) 104089031$

Please send questions and/or remarks of nonscientific nature to driessen@tinbergen.nl.

Most TI discussion papers can be downloaded at http://www.tinbergen.nl. 


\title{
ON THE STRATEGIC USE OF FOCAL POINTS IN BARGAINING SITUATIONS
}

\author{
Maarten C.W. Janssen ${ }^{1}$ \\ Erasmus University Rotterdam and Tinbergen Institute
}

\begin{abstract}
This paper argues that the notion of focal points is important in understanding bargaining processes. Recent literature confines a discussion of the usefulness of the notion to coordination problems and when bargaining experiments result in outcomes that are inconsistent with a straightforward interpretation of economic theory, some notion of 'fairness' is invoked. This paper uses symmetry requirements to formalize the notion of focal points. By doing so, it explains the focality of equal split division and it re-interprets recent experimental evidence in bargaining games. Experimental economists should try to empirically disentangle the importance of focal points from other explanatory factors (such as fairness). One way to do so, would be to study modal (instead of average) responses more systematically. Future theoretical research should focus on the strategic implications of proposing a frame (focal point) to conceive of the bargaining problem.
\end{abstract}

Key Words: Bargaining, Game Perceptions, Focal Points

JEL code: C78, C91

PsycINFO codes: 2360, 3020, 3040

\footnotetext{
${ }^{1}$ Correspondence Address: Erasmus University Rotterdam and Tinbergen Institute, Postbus 1738, 3000 DR Rotterdam, The Netherlands, e-mail: janssen@few.eur.nl. I thank Vince Crawford, Robert Dur, Armin Falk, Werner Güth, Hans Haller and two anonymous referees for very helpful comments on an earlier version.
} 


\section{Introduction}

In contrast to mainstream approaches in game theory, Schelling (1960) forcefully argued that individuals in everyday life situations frequently use information contained in the labels of strategies to decide which strategies to choose. Schelling showed how the use of mutually recognized or recognizable signs (labels) may help players to reach a mutually beneficial outcome. He introduced the term 'focal point' to account for this use of labels.

Schelling used games of pure coordination mainly as a means to illustrate how focal points may be used. However, Schelling (1960) ultimately was more concerned with bargaining than with games of pure coordination. ${ }^{2}$ The fact that he regarded focal points also to be of importance in bargaining situations (“mixed-motive games”) stems from the fact that there is a coordination aspect present in each and every bargaining problem in the sense that "parties recognize that there is a wide range of outcomes preferable to both of them over no agreement at all” (Schelling, 1960, pp. 101)). Within this set of mutually beneficial outcomes, there is conflict of interest. He continues by arguing “[T]he psychic moment of 'mutual perception’ that can be verified as real and important in the tacit [bargaining] case has a role to play in the analysis of explicit bargaining. Coordination of expectations is the role.” (p. 101). Other passages stressing the similarities between tacit and explicit bargaining in terms of focal points can be found on pages 72-4 of Schelling (1960).

Unfortunately, most of the later literature on focal points has restricted itself to coordination games. The more recent coordination literature on focal points can be divided into three strands. The experimental literature has basically confirmed the results of Schelling's informal experiments and tested some of the more recent formal theories (see, e.g., Mehta et al. (1994) and Bacharach and Bernasconi (1997)). The evidence suggests that individuals are indeed able to successfully use differences in strategy labels to coordinate their actions. Earlier philosophical literature, on the other hand, emphasized the difficulties of making the notion of focal points consistent with

\footnotetext{
${ }^{2}$ Of course, I do not want to argue that Schelling's contributions to the bargaining literature are confined to the notion of focal points only. For a recent overview discussing the width of Schelling's work, one may wish to consult http://nobelprize.org/economics/laureates/2005/ecoadv05.pdf.
} 
the rationality notion used in game theory without denying the empirical validity (see, e.g., Gautier (1975), Gilbert (1990)). Finally, more recent theoretical literature, initiated by Bacharach $(1991,1993)$ and Crawford and Haller (1990) and later by Casajus (2000), Sugden (1995) and Janssen (2001)), incorporates the notion of focal points into a more formal theory of games by redefining (but not eliminating) the rationality notion. The present paper shows how some of the ideas expressed in this more theoretical literature can be extended so that they are applicable to bargaining situations.

The main line developed in this paper is that players use their knowledge of the bargaining game and the perceived common background to look for different possibilities to describe the game in ways that potentially may also be recognized by other player(s). If players cannot find a vocabulary that they think is mutually recognizable in which they can distinguish between some strategies, then these strategies are symmetric with respect to each other. Players must treat these 'undistinguishable' strategies in a symmetric way. Moreover, and this may be important in bargaining situations, players may not be able to usefully distinguish between the positions they have in the game. For example, in symmetric games it may be unclear to the players whether they are Column choosers or Row choosers. In this case, players must consider symmetric strategy combinations only. The symmetry requirements thus imposed create constraints on the sets of strategies players may possibly choose. The constraints imposed by these frames help players to find mutually beneficial bargaining outcomes.

The main point the paper wants to make is that focal points deserve serious attention in the bargaining literature. To my knowledge, there is only one other paper (Binmore et al. (1993)) explicitly dealing with the notion of focal points in bargaining. That paper is, however, experimental in nature and deals with the evolution of conventions in a specific set-up. Moreover, the present paper makes a first attempt to use focal points to explain the frequently observed equal-split division of the surplus. The paper re-interprets experimental evidence on ultimatum bargaining games and a recent experiment by Falk and Kosveld (2004) and argues that these results may well be (partly) driven by focal point considerations rather than by fairness considerations only. One way to disentangle focal points and fairness considerations, so this paper 
argues, is by investigating differences between modal and average responses more carefully than is typically done in the experimental literature.

The rest of the paper is structured in the following way. The next Section provides an example of a coordination game that enables us to informally describe the main concepts used in this paper. The notion of action symmetry and player symmetry are more formally introduced in Section 3. Section 4 then shows that in quite a few bargaining situations, a 50-50 split can be rationalized in terms of the concepts introduced. Section 5 continues in a slightly more speculative fashion by arguing that some of the recent behavioural literature on ultimatum bargaining games can be reinterpreted in terms of focal point considerations. Section 6 concludes with some suggestions for further research. It is argued that experimental research must attempt to empirically disentangle focal points from other potentially important factors explaining results found in the laboratory. Theoretical research may focus on the way labels may be used strategically, an idea that is underdeveloped so far.

\section{A Simple Example of a Coordination Game}

To illustrate the main ideas consider the following example. Suppose individuals 1 and 2 have independently of each other to choose one out of three blocks. The players do not observe the choice made by the other and cannot communicate. All observable characteristics of the blocks that are mutually recognized are identical apart from their colour, i.e., locations and/or other characteristics are scrambled to such an extent that players cannot use these to coordinate their actions. One block is blue, the other two are green. Inside each block, a number indicates the identity of the block. Each of the three blocks contains a different number and the number is unobservable for the players that have to make choices. Only after the choice is made, the block is opened to determine which block a player has chosen. If, and only if, the players choose the same block, identified by the number inside, the players are rewarded. That is, the interaction structure is one of pure coordination. Which block should a player choose?

The natural solution seems to be that both players choose the blue block. The reason is as follows. The players know that the only way they receive a reward is by picking 
the same block as the other player. They therefore have to look for a clue that can be recognized by both. The example is structured in such a way that the only difference between the blocks is their colour. Even though there are three blocks, it seems therefore that the players conceive of the game as one of choosing between blue and green. If a player chooses green, she has no reason to distinguish between the two green blocks. She therefore will treat the two green blocks in a symmetric way in any choice she will make. Given this symmetry, it remains uncertain what the pay-off of choosing green is. Even if both players choose green, there is a probability (equal to a half, if symmetry is respected) that they do not choose the same block. However, if both players choose blue, then the reward is maximal and certain. Intuitively, therefore, the players should choose blue.

The two principles that are used in this paper to arrive at this solution are the Principle of Insufficient Reason (PIR) and, what I will call, the Principle of Individual Team Member Rationality (PITMR). PIR basically says that a rational choice cannot discriminate between two strategies if they have the same characteristics. At a more formal level, the principle is implemented by using the idea that an attribute induces a partitioning of the strategy set into equivalence classes and that a rational choice treats members of the same cell symmetrically. In this particular example, colour divides the strategy set into a cell with one blue block and a cell with two green blocks. A strategy that respects PIR gives equal probability to the two green blocks. The pay-off matrix as perceived by the players (one choosing rows, the other choosing columns) can thus be represented as in Table 1, where the reward is normalized to be equal to 1 .

\begin{tabular}{|l|l|l|}
\hline & Choose Blue & Choose Green \\
\hline Choose Blue & 1,1 & 0,0 \\
\hline Choose Green & 0,0 & $1 / 2,1 / 2$ \\
\hline
\end{tabular}

Table 1. The transformed 'choosing a block' game

PITMR says that if in the class of strategy combinations that respect PIR there is a unique strategy combination that is Pareto-optimal, then individual players should play their part of that strategy combination. Such a unique Pareto-optimal strategy combination forms inevitably a Nash equilibrium of the transformed game. The notion of PITMR is identical to Luce and Raiffa's (1957, pp. 107) "solution in the 
strict sense”. The uniqueness requirement is added to avoid confusion among the players (see also the discussion in Schelling (1960, pp. 291ff). PITMR is similar to the criterion of pay-off dominance (as proposed by Harsanyi and Selten (1988) and further analyzed by Colman and Bacharach (1997), among others), the principle of coordination (as proposed by Gauthier (1975)) and the criterion of collective rationality (as proposed by Sugden (1991)). The main difference with most of these notions is the uniqueness requirement. In the present case, the class of mixed strategy combinations that respect PIR can be parameterized by $\left\{\left(p_{1 b}, p_{1 g}, p_{1 g}\right)\left(p_{2 b}, p_{2 g}, p_{2 g}\right)\right\}$, with $p_{1 b}+2 p_{1 g}=1$ and $p_{2 b}+2 p_{2 g}=1$, where $p_{1 b}\left(p_{2 b}\right)$ represents the probability with which individual 1 (2) chooses the blue block and $p_{1 g}\left(p_{2 g}\right)$ represents the probability with which individual 1 (2) chooses a green block. It is easy to see that the unique Pareto-optimal strategy combination that satisfies PIR is $\{(1,0,0),(1,0,0)\}$. Thus, the combination of the two principles explains the intuitive idea that players will choose the blue block.

The example exploits the fact that if players cannot distinguish between certain strategies, these strategies should be treated symmetrically. Alternatively, it can be argued that players cannot distinguish between the different roles (Row or Column chooser) they may have in playing the game. Therefore, in addition to the symmetry implied by the attributes of the strategies, another type of symmetry implies that players really should play identical strategies. Symmetry with respect to the positions of players is not used in the example above, and if we would consider it, the argument would not change fundamentally. In the bargaining examples we consider below, symmetry across players' positions is, however, crucial.

The above example is special in that it only investigates the consequences of the strategies being different along one attribute (or dimension), namely colour. Other difficulties arise when strategies can be distinguished along two or more dimensions. In this case, players can conceptualize playing the game in different ways. Each of these conceptualizations induces a different view of how the game can be played. Depending on the conceptualization (frame), different strategies open up and different pay-offs can be realized. Bacharach (1993) and later Janssen (2001) have introduced the idea of Variable Universe Games to analyze the way players can choose which 
frame to adopt in coordination games. Part of the strategic behaviour of players is which conceptualization to choose. In bargaining situations, players may further try to impose a certain frame on how to play the game. Equal split is usually a convincing focal point (and we will argue below why), but what to split equally depends very much on the perceived frame (conceptualization). This part of the decision problem is not analyzed in traditional game theory, because a particular conceptualization is imposed on the players when the game theorist specifies the strategy space of the game.

The principles of PITMR and PIR are not uncontroversial. Goyal and Janssen (1996) discuss the similarities and differences between the different concepts. Bacharach (1991, 1993) also employs the notion of PIR. A discussion of the pros and cons of PIR and PITMR can be found in Janssen (2001), among others.

\section{Symmetry}

In this section, I give a more formal description of the types of bargaining situations we consider and the way I introduce, more formally the notion of action symmetry and position (or players') symmetry. These definitions follow to a large extent the definitions given in Crawford and Haller (1990). Experimental evidence on the framework presented here is provided by Blume (2000) and Blume and Gneezy (2000).

Two players, denoted by 1 and 2, play a bargaining game where the bargaining is how to divide the gains from trade. These gains are normalized to be equal to 100 . Given a certain frame $(F)$ of the game Player 1's strategies are called the Row strategies and denoted by $R(F)$; player 2 chooses column strategies denoted by $C(F)$. Unlike Janssen (2001) I only consider situations where both players have the same frame. The reason framing is discussed at all is that in some bargaining situations I consider, one of the players has the possibility to offer a certain way the bargaining problem is to be framed. Depending on the frame proposed a different bargaining situation emerges. As the relevance of strategic framing decisions has not been discussed in the literature before, I consider for simplicity only bargaining problems where a framing decision can be imposed by one of the players so that in the 'real' bargaining game, both 
players adopt the same frame. Hence, a pre-bargaining stage concerning the frame to be adopted is excluded from the analysis. In case examples are discussed in which framing is not relevant, the dependence of $R$ and $C$ on $F$ is simply dropped. The payoffs for the two players when 1 chooses strategy $r \in R(F)$ and 2 chooses strategy $c \in C(F)$ are denoted by $\pi_{i}(r, c), i=1,2$. If $r$ and $c$ are such that an agreement is reached, then $\pi_{1}(r, c)+\pi_{2}(r, c)=100$. The game structure is assumed to be common knowledge.

There are two types of symmetry I would like to discuss. A first type of symmetry, description symmetry, depends on the frame that is adopted. Given a certain frame $F$, description symmetry says that a player should assign the same probability to all actions that are descriptively identical under that frame. Description symmetry is implied by PIR. It is the type of symmetry used in the example of Section 2 to argue that players in that example should choose a strategy that gives equal probability to the two green objects.

Position symmetry, on the other hand, refers to the symmetric position players in a bargaining situation may have vis-à-vis each other. Of course, from one perspective one may argue that players in most bargaining situations are not symmetric to each other, for example, simply because one player wants to sell something, while the other wants to buy. However, if adopting the frame of bargaining over a pie (common surplus) is legitimate, then it is obvious that the two players are in a symmetric position vis-à-vis each other if there is no exogenously imposed rule creating an asymmetry between the players. This is true even if the size of the pie is uncertain as both players have incomplete information concerning the reservation price of the other player.

I define position symmetry in the following way. Players' positions are symmetric with respect to each other if interchanging them yields (possibly after permuting rows and columns) the same pay-off matrix (for more details, the reader is referred to Crawford and Haller, 1990, pp. 579). Position symmetry tells players to choose identical strategies if players’ positions are symmetric. 
An attainable strategy combination is one that respects description and position symmetry whenever they apply. PITMR then says that if there is a unique Paretooptimal attainable strategy combination that is such that individually players do not have an incentive to deviate from it, then players choose their part of this strategy combination. The requirement "that individually players do not have an incentive to deviate from it" is added to avoid that the solution concept tells players to cooperate if they play a Prisoner's Dilemma game.

Reading some parts of Schelling (1960), the reader may get the impression that the author is opposed to the imposition of mathematical symmetry requirements. For example, he argues that "The uncertainty that can usually be presumed to exist about each other's value systems also reduces the usefulness of the concept of mathematical symmetry. Mathematical symmetry cannot be perceived if one has access to only half of the relevant magnitudes.” (p. 117-8). Our discussion gets around this problem by considering the case where either both players know each others' reservation prices, or alternatively, and arguably more realistic, where both players only know their own reservation price and they are symmetrically (un)informed about the reservation price of the other player.

\section{The Focality of Equal Split}

In this section I consider a specific bargaining game, the so-called Nash demand game, and analyze it with the concepts defined in the previous section. Two players choose independently of each other a natural number between 0 and 100, representing the share (in percentage terms) of the surplus (cake) that will be divided between them. If the sum of the shares sums up to a number smaller than or equal to 100 , then their claims are compatible and they both receive the share they mentioned. If the sum is larger than 100, the claims are incompatible and nobody receives anything. The game can be represented as in Table 2 below, where player 1 chooses rows and player 2 chooses columns. 


\begin{tabular}{|l|l|l|l|l|l|l|l|l|}
\hline & 0 & 1 & 2 & 3 & $\ldots$ & 98 & 99 & 100 \\
\hline 0 & 0,0 & 0,1 & 0,2 & 0,3 & $\ldots$ & 0,98 & 0,99 & 0.100 \\
\hline 1 & 1,0 & 1,1 & 1,2 & 1,3 & $\ldots$ & 1,99 & 1,99 & 0,0 \\
\hline 2 & 2,0 & 2,1 & 2,2 & 2,3 & $\ldots$ & & 0,0 & 0,0 \\
\hline 3 & 3,0 & 3,1 & 3,2 & 3,3 & $\ldots$ & 0,0 & 0,0 & 0,0 \\
\hline$\ldots$ & $\ldots$ & $\ldots$ & $\ldots$ & $\ldots$ & $\ldots$ & 0,0 & 0,0 & 0,0 \\
\hline 98 & 98,0 & 98,1 & 98,2 & 0,0 & 0,0 & 0,0 & 0,0 & 0,0 \\
\hline 99 & 99,0 & 99,1 & 0,0 & 0,0 & 0,0 & 0,0 & 0,0 & 0,0 \\
\hline 100 & 100,0 & 0,0 & 0,0 & 0,0 & 0,0 & 0,0 & 0,0 & 0,0 \\
\hline
\end{tabular}

Table 2. A discrete version of the Nash demand game where the first Column (Row) represents the share the first (second) player claims.

This game has many Nash equilibria. Any combination $(r, c)$ with $r+c=100$ forms a Nash equilibrium. There is, however, one solution that seems to stick out, namely $(50,50)$ and it would seem that many real world players would go for this particular choice. Loosely speaking, the reason seems to be something like the following. Any strategy combination $(j, 100-j), j=1, \ldots, 100$ is as good as any other. Due to the strategic uncertainty implied by the fact that the two players choose independently of each other, the players do not have any reason to discriminate between $j$ and 100-j. Even if they somehow think that together they will choose the strategy combination where one plays $j$ and the other chooses $100-j$, they have no way to know who will play which strategy ( $j$ or $100-j$ ). The problem implied by this form of strategic uncertainty is not present if, and only if, $j=100-j$, or $j=50$.

Although there are other (mostly axiomatic) solution concepts, most notably the Nash bargaining solution, available in the literature that select the 50-50 split as the outcome of the game, there does not exist a formalization of the above more behavourial intuition. Using the notions expressed in the previous section, the intuition can be formalized as follows. Let us denote by $p_{i j}$ the probability that player $i$ chooses number $j$. It is clear that without any additional frame, all actions are different from one another and therefore that description symmetry does not invoke any restrictions upon the strategies players can choose. It is easy to see, however, that the 
positions of the two players are symmetric: interchanging the Row and Column position does not change the players' pay-offs. Position symmetry then implies that $p_{1 j}=p_{2 j}=p_{j}$. Given this restriction on the attainable mixed strategy combinations, the expected pay-off to each of the players equals $\sum_{j=0}^{100} \sum_{k=0}^{100-j} j \cdot p_{j} p_{k}$. Given the non-negativity constraints $p_{j}>0$ and $\sum_{j=0}^{100} p_{j}=1$, one can show that this expression reaches a (unique) maximum for $p_{50}=1$ and $p_{j}=0$ for $j \neq 50$. Thus, the optimal attainable strategy combination is for both players to choose the strategy 50 for sure. As both players choosing 50 is a Nash equilibrium, it is easy to see that no one individually has an incentive to deviate from choosing this strategy. PITMR can then be applied to argue that both players individually will choose to play 50 .

Another way to derive the focality of the 50-50 split is to say that players can think of the following 'equality' frame: they either choose an equal division, or they choose an unequal division. If they choose an unequal division, description symmetry under this frame tells players to give equal probability to any strategy that proposes an unequal division. Given this `equality’ frame, the pay-off matrix reduces to the one given in Table 3. (The pay-off of choosing unequal division are calculated as follows. If the other player chooses equal division, then the pay-off of choosing unequal division is $\sum_{j=0}^{49} j / 100$. If the other player chooses unequal division, then this pay-off equals $\left(\sum_{j=0}^{49} j(100-j)+\sum_{j=51}^{100} j(100-j+1)\right) / 10000$. $)$

\begin{tabular}{|l|l|l|}
\hline & Choose Equal Division & Choose Unequal Division \\
\hline Choose Equal Division & 50,50 & $25,12.375$ \\
\hline Choose Unequal Division & $12.375,25$ & $16.7925,16.7925$ \\
\hline
\end{tabular}

Table 3. A framed version of the Nash demand game.

It is easy to see from Table 3 that given this frame, choosing Equal Division is a dominant strategy equilibrium.

The original bargaining game analyzed in this Section can be played in many different variations. For example, it can be argued that if both players' claims are incompatible they have the possibility to renegotiate in a next period. As the symmetric game then 
repeats itself one may argue that whatever the claims made at that stage, players will receive the same expected pay-off in the continuation game. Moreover, if players discount future pay-offs, there is a cost associated with not reaching an immediate agreement. Replacing the pay-off of $(0,0)$ by $(x, x)$ with $x<1 / 2$ implies that the expected pay-off formulae will change into $\sum_{j=0}^{100} \sum_{k=0}^{100-j} j \cdot p_{j} p_{k}+\sum_{j=0}^{100} \sum_{k=100-j+1}^{100} x \cdot p_{j} p_{k}$. It is easy to see that this change in pay-offs does not effect the remainder of the analysis: 50 remains the focal point.

Alternatively, one may argue that if the sum of the shares that are claimed by the players is smaller than 100, they may still sit down at a later moment to divide the remaining surplus. Again, if these future pay-offs are discounted, the above symmetry principles claim that players will split the surplus equally and choose 50.

\section{Focal Points in Ultimatum Games}

The bargaining example analyzed in the previous section is, of course, just one highly simplified structure in which bargaining takes place. Real life bargaining problems contain many other elements that are not captured by this example. In the present section I will discuss some examples of bargaining games that contain elements of commitment and threat not present in the example analyzed in the previous section. In particular, I will discuss a series of experimental results on ultimatum games and the recent experiments by Falk and Kosveld (2004).

The ultimatum bargaining game plays a central role in the bargaining literature and has attracted a lot of attention from theoretical and experimental economists alike. The game assigns the right to make an offer (a share of the surplus to be divided) to one of the players (the Proposer) and gives the second player (the Receiver) only the right to accept or reject this proposal. If the proposal is accepted, it is implemented; if it is rejected, none of the players gets anything. The ultimatum game has many Nash equilibria. Basically, any division of the surplus can be represented as the outcome of a Nash equilibrium (like in the game of the previous section). However, because of the sequential nature of the game the notion of subgame perfection can be invoked to 
reach the conclusion that the Proposer should propose a very small fraction of the surplus to the receiver (or even nothing) and that the receiver should accept every proposal that is made. Of course, this solution implicitly assumes that (i) people are self-interested and that (ii) the structure of the pay-offs is common knowledge among the players. If one of these conditions fails, the Proposer may rationally propose more to the Receiver than a very small share.

A wide range of papers (see, e.g., the initial paper by Güth et al. (1982) being the first and Hoffman et al. (1994), and more recently Camerer (2003, chapter 2) for an overview and discussion of different contributions) provides experimental evidence showing that Proposers actually propose much more than what the subgame perfect equilibrium suggests Proposers should propose and that Receivers frequently reject marginal proposals. In fact, the modal offer is almost always to divide the surplus equally.

One way to explain this pattern is to introduce the idea that individuals have a preference for 'fairness'. The interpretation of fairness as inequity aversion (see, Fehr and Schmidt, 1999) has come to dominate a large literature. Without denying the usefulness of explaining equal split outcomes in terms of fairness considerations, I would like to propose an alternative explanation based on focal points. The alternative explanation starts by recognizing that even in the ultimatum game there are multiple Nash equilibria and that players face a selection problem. Subgame perfection is one mechanism selecting among the many equilibria. Subgame perfection is based on the fact that the sequencing of actions breaks the symmetry between players and favors the player making the proposal. An explanation in terms of focal points assumes that despite the 'objective asymmetry' players perceive of the interaction in a symmetric way. ${ }^{3}$ Depending on the framing of the ultimatum game, players may readily translate the game into their own vocabulary in something like the following: "One of us is randomly assigned the role of Proposer. Independent of whether I or my game mate will be assigned this role, I should think of a number representing both the share I will propose to my game mate if I have the role of Proposer and the minimum share I will

\footnotetext{
${ }^{3}$ Perceiving the ultimatum game as symmetric may seem farfetched. However, others (Pull, 2003, and Selten, 2000) have also offered this as a potential explanation for observed behaviour. Later in the paper I present experimental evidence suggests players may actually perceive the game in a symmetric way.
} 
accept if I play the role of Receiver”. It is clear that if players perceive the ultimatum game in this way, then the subjective game the players are playing is a kind of coordination game described in Table 4 below, where each cell contains the expected pay-off of the players. Using position symmetry or description symmetry in the equality frame then reveals that choosing 50 is the focal point.

\begin{tabular}{|c|c|c|c|c|c|c|c|c|}
\hline & 0 & 1 & 2 & 3 & $\ldots$ & 98 & 99 & 100 \\
\hline 0 & 50,50 & $1 / 2,491 / 2$ & 1,49 & $11 / 2,481 / 2$ & $\ldots$ & 49,1 & $491 / 2,1 / 2$ & 50,0 \\
\hline 1 & $491 / 2,1 / 2$ & 50,50 & 1,49 & $11 / 2,481 / 2$ & $\ldots$ & 49,1 & $491 / 2,1 / 2$ & 50,0 \\
\hline 2 & 49,1 & 49,1 & 50,50 & $11 \frac{1}{2}, 481 / 2$ & $\ldots$ & 49,1 & $491 / 2,1 / 2$ & 50,0 \\
\hline 3 & $481 / 2,11 / 2$ & $48^{1 / 2,11 / 2}$ & $481 / 2,11 / 2$ & 50,50 & $\ldots$ & 49,1 & $491 / 2,1 / 2$ & 50,0 \\
\hline$\ldots$ & $\ldots$ & $\ldots$ & $\ldots$ & $\ldots$ & $\ldots$ & 49,1 & $491 / 2,1 / 2$ & 50,0 \\
\hline 98 & 1,49 & 1,49 & 1,49 & 1,49 & 1,49 & 50,50 & $491 / 2,1 / 2$ & 50,0 \\
\hline 99 & $1 / 2,491 / 2$ & $1 / 2,491 / 2$ & $1 / 2,491 / 2$ & $1 / 2,491 / 2$ & $1 / 2,491 / 2$ & $1 / 2,491 / 2$ & 50,50 & 50,0 \\
\hline 100 & 0,50 & 0,50 & 0,50 & 0,50 & 0,50 & 0,50 & 0,50 & 50,50 \\
\hline
\end{tabular}

Table 4. Ultimatum Bargaining Under a Veal of Symmetry.

Is there any experimental evidence that players may perceive of the ultimatum game in a symmetric way like the perception described above? I think the evidence presented in Hoffman et al. (1994) may be re-interpreted in this way. Hoffman et al. (1994) conduct many different versions of the ultimatum game experiment, two of which are important for our purposes. In one treatment, they have the Proposer being determined at random, whereas in another treatment the players first play a contest (a current events quiz) and the winner of the contest gets the right to play the role of Proposer. In the random assignment treatment (mimicking the perception described above) a little more than $50 \%$ of the Proposers chose to offer an equal split. Moreover, in that treatment some (albeit insignificant number of) unequal offers were rejected. In the contest treatment, only $10 \%$ of the proposals split the surplus equally and all offers were accepted. In both treatments, the 'objective' bargaining game is asymmetric: all bargaining power is in the hands of the Proposer. However, randomly determining the Proposer strengthens the symmetry perception of players, which makes them propose an equal split. Winning a Contest provides the Proposer (and the Receiver) with a reason to break the symmetry and the focal point of a 50-50 split. In the contest treatment, the focal point of equal split is (at least) partly replaced by an 
equally pervasive frame (perspective) that "the winner deserves more”. As this perspective does not have a clear focal point, offers seem to be much more spread out. In a similar vein, Güth and Tietz (1986) presents an experiment in which the right to be the Proposer is auctioned. They also find that the original surplus is not split equally. Interestingly, however, they also find that the proposer does not keep everything to himself, but rather decides on a split of the surplus taking account of the amount of money paid in the auction.

Another way to think of explaining equal split divisions in ultimatum games in terms of focal points is by introducing uncertainty concerning relevant pay-off functions. If the Proposer is uncertain about the pay-off function of the Receiver, i.e., the minimal share that is still acceptable to the Receiver, then the following observation taken from Schelling (1960, p. 111-2) seems relevant:

In fact, a focal point for agreement often owes its focal character to the fact that small concessions would be impossible, that small encroachments would lead to more and larger ones. One draws a line at some conspicuous boundary or rests his case on some conspicuous principle that is supported mainly by the rhetorical question, "if not here, where?"

There is not just a marginal difference between a 50-50 split and a 51-49 split. The first is an equal division, the second is not. Above I have formalized this idea using description symmetry in an 'equality' frame. The importance of this difference is corroborated in an experiment (Güth et al., 2001). In this paper, results of three experiments are reported in which equal split in one experiment is replaced by nearly equal split in the two other experiments. The "fair" offer is chosen much more often if an exact equal split can be chosen.

The large experimental literature goes into some depth in trying to explain the observed deviations from standard economic theory for Proposers and Receivers separately. The common idea here is that it is probably most difficult to reconcile observed rejection rates (see, e.g., Falk et al. (2003)). Above I have, however, adopted a framework to formalize focal points that is based on the notion that players conceive of their situation in a symmetric way and that a focal point really is a rule players are looking for that helps them overcome a coordination problem or an equilibrium 
selection problem. Focal points have then, by their very nature, not very much to say on the single agent decision problem, which the Receiver's decision situation in a sense is.

In order to empirically distinguish between fairness considerations and focal point considerations, one may try to exploit differences in behavior between Proposers and Receivers along the lines sketched above. Another way to discriminate between focal point considerations and fairness considerations is to exploit differences between modal responses and average responses in an experiment. It seems that fairness considerations can be more easily traded off against bargaining power considerations: proposing a 60-40 split can be easily rationalized by arguing that a Proposer cares quite a bit about fairness, but that he also wants to exploit his own bargaining power to some extent. And the same applies to a 61-39 split. As focal points very much center on rules that are easily recognized by players, however, such a trade-off is not easily conceivable for focal points. Players either follow a rule or not. If focal point considerations are important for many players, one should see therefore a modal response that is chosen by many players.

Recently, Falk and Kosfeld (2004) have conducted a fascinating experiment in which a variation on the ultimatum bargaining game was played. Instead of being in the position to accept or reject an offer, the Receiver in their set-up was in the position to choose whether or not she wanted to impose a minimum on the amounts to be offered to her. Any offer that was eventually made was automatically accepted. There were also some other, less important, differences to the standard ultimatum game. First, the game was cast in terms of a principal-agent framework. Any amount $x$ that the agent proposes not to keep for himself is doubled before it is given to the Principal (Receiver). The total amount that the agent disposes of equals 120 so that the final pay-offs are $120-x$ for the agent and $2 x$ for the principal. Thus, if the agent proposes $x=40$, then both players receive a pay-off of 80 . In different treatments, this minimum bound $\underline{x}$ was specified ex ante to be equal to 5, 10 and 20, respectively. The main result they find is that if Principals impose a minimum bound, then agents choose much lower offers than in case they have an unrestricted choice set. Moreover, most Principals decide not to restrict the choice set. Falk and Kosfeld (2004) interpret their findings in terms of 'trust'. If agents feel they are trusted (i.e., no lower bound is 
imposed) they are ready to give more. Imposing a lower bound is a signal of distrust that is returned by a low offer. The title nicely reflects this interpretation: "The Hidden Cost of Control”.

Not denying the plausibility of this interpretation and the discussion of different possible motivations they provide, I would like to offer another interpretation based on the notion of focal points. Looking at Figure 1 in Falk and Kosfeld (2004) it turns out that in case no minimum bound is imposed, the modal offer is 40 . Depending on the treatment, this offer is made by $25-40 \%$ of the Proposers. Apparently, in the absence of minimum bounds agents make such a proposal that both players receive the same pay-off. From Schelling's focal point perspective of "if not there, where?" this behavior is easily understood: symmetry or equality seems to be the only or certainly the most outstanding principle to us in deciding what to offer.

In the case a minimum bound was imposed, this situation changes dramatically. With minimum bounds, $50 \%$ or more of the agents proposes to offer an amount exactly equal to the minimum bound. My interpretation of this result is that when introducing a minimum bound, the Principal offers in fact another frame to the agent to think about the problem what to offer: "I give him what he asked for". By requiring a minimum offer, the Principal implicitly offers a new answer to the question "if not there, where?” Understanding the strategic effect of creating such a new focal point, the Principal realizes that it is not in his interest to propose such a frame to think of what to offer and that proposing such a frame is actually a bad strategic move. In the experiment, most Principals understand the logic so that in fact they do not create a new focal point.

\section{Discussion and Conclusion}

The notion of Focal Point is rarely used in recent theoretical or experimental research on bargaining. The unimportance attached to focal points becomes apparent once it is realized that modal responses are not presented. Keeping the notion of focal points in mind, there is a sharp (and not a marginal) difference between proposing a 50-50 split or a 51-49 split of the surplus: A 50-50 split is an equal division, all other proposals 
are unequal! Experimental research reports average proposals, median proposals, or that more than $x \%$ of the proposals gives more than $y \%$ to the Receiver. The fact that the modal proposal is often 50-50 does not receive attention. If focal points are taken seriously, it is modal (and not median or average) proposals that should be focussed on as explained in the previous section.

This paper's main message is that focal points are worth studying in the context of bargaining problems. Mutual expectations about reservation prices are crucial in bargaining. Coordination of these expectations brings about the possibility of multiple equilibria. Invoking the notion of subgame perfection is one way to select among equilibria; focussing on focal points is another. Without other points to coordinate expectations, focal points in bargaining games usually center around equal division of the surplus.

An interesting issue for future research is how focal points can be used strategically by players in bargaining situations. One way to consider is that certain actions create new points of focality, such as in the experiments reported by Falk and Kosfeld. In their experimental game, the pay-offs are such that it is not a wise strategy to create such a focal point. In other games, it may actually be the other way around and that players do have incentives to create and impose new ways to think of the bargaining situation. One such a way is to create alternative frames of thinking about the surplus that is to be divided. Another line of research should focus on ways to experimentally disentangle focal point explanations for bargaining outcomes from other explanations. The difference between modal and average responses should play a key role here as many players should choose the modal response if focal points are important, whereas this is not the case for fairness-based explanations.

\section{References}

Bacharach, M. 1991. Games with Concept-Sensitive Strategy Spaces. Oxford University, mimeo.

Bacharach, M. 1993. Variable Universe Games; in Binmore, K. Kirman, A. and Tani, P. (eds.). Frontiers of Game Theory. Cambridge (Mass.): MIT Press, pp. 255-75. 
Bacharach, M. and Bernasconi, M. 1997. An experimental study of the variable frame theory of focal points, Games and Economic Behavior 19: 1-45.

Binmore, K. J. Swierzbinski, S. Hsu and C. Proulx. 1993. Focal Points and Bargaining. International Journal of Game Theory 22: 381-409.

Blume, A. 2000. Coordination and Learning with a Partial Language. Journal of Economic Theory 95 (1): 1-36.

Blume, A. and U. Gneezy. 2000. An Experimental Investigation of Optimal Learning in Coordination Games. Journal of Economic Theory 90: 161-172.

Camerer, C. 2003. Behavorial Game Theory: Experiments on Strategic Interaction. New York: Princeton University Press.

Casajus, A. 2000. Focal Points in Framed Strategic Forms. Games and Economic Behavior 32: 263-91.

Colman, A. and M. Bacharach (1997), Payoff dominance and the Stackelberg heuristic. Theory and Decision 43: 1-19.

Crawford, V. and H. Haller. 1990. Learning How to Cooperate: Optimal Play in Repeated Coordination Games. Econometrica 58: 571-95.

Falk, A., E. Fehr and U. Fischbacher. 2003. On the Nature of Fair Behavior. Economic Inquiry 41 (1), pp. 20-6.

Falk, A. and M. Kosfeld. 2004. The Hidden Cost of Control. American Economic Review (forthcoming).

Fehr, and K. Schmidt. 1999. A Theory of Fairness, Competition and Cooperation. The Quarterly Journal of Economics 114, pp. 817-868

Fisher, S., W. Güth, W. Müller and A. Stiehler. 2003. From Ultimatum to Nash Bargaining: theory and Experimental Evidence, mimeo.

Gauthier, D. 1975. Coordination. Dialogue 14: 195-221.

Gilbert, M. 1990. Rationality, Coordination and Convention. Synthese 84: 1-21.

Goyal, S. and M. Janssen. 1996. Can we Rationally Learn to Coordinate? Theory and Decision 40: 29-49.

Güth, W., S. Huck and W. Müller. 2001. The Relevance of Equal Splits in Ultimatum Games. Games and Economic Behavior 37, pp. 161-169.

Güth, W., R. Schmittberger. and B. Schwarze. 1982. An Experimental Analysis of Ultimatum Bargaining. Journal of Economic Behavior and Organization 3, pp. 347369. 
Güth, W. and R. Tietz. 1986. Auctioning Ultimatum bargaining positions-how to act rational if decisions are unacceptable? In R.W. Scholz (ed.). Current Issues in West German Decision Research, pp. 173-85. Frankfurt: Verlag Peter Lang.

Harsanyi, J. and R. Selten. 1988. A General Theory of Equilibrium Selection in Games. Cambridge, Mass.: MIT Press.

Hoffman, E., K. McCabe, K. Shachat and Smith, Vernon L. 1994. Preferences, Property Rights, and Anonymity in Bargaining Games. Games and Economic Behavior 7(3): 346 -80.

Huyck, J. Van, R. Battalion, S. Mathur, P. Van Huyck and A. Ortmann. 1995. On the Origin of Convention: Evidence from Symmetric Bargaining Games. International Journal of Game Theory 24: 187-212.

Janssen, M. 2001. Rationalizing Focal Points. Theory and Decision, vol. 50, pp. 119148.

Mehta, J., C. Starmer and R. Sugden. 1994. Focal Points in Pure Coordination Games: An Experimental Investigation. Theory and Decision 36: 163-185.

Pull, K. 2003. Ultimatum games and wages: Evidence of an `implicit bargain'? Schmalenbach Business Review, 55: 161-171.

Schelling, T. 1960. The Strategy of Conflict. Cambridge, Mass.: Harvard UP.

Selten, R. 2000. Thünen \{Vorlesung: Eingeschrankte Rationalitat und Okonomische Motivation. Zeitschrift fur Wirtschafts- und Sozialwissenschaften, 9 (special issue):129-157.

Sugden,R. 1995.Towards a Theory of Focal Points. Economic Journal 105: 533-50. 